\title{
EDUTIC: Plataforma de Aprendizaje Adaptativo - Transformando la Educación Básica Regular
}

\author{
Alexander Ocsa ${ }^{1}$, Gustavo Suero $^{1}$, José Herrera ${ }^{1}$, Klinge Villalba ${ }^{1}$ \\ ${ }^{1}$ Universidad Nacional de San Agustín \\ Catedra Concytec en TICs - CITEC - UNSA \\ Arequipa - Perú \\ \{aocsa.cs,tsuero.s, jherreraq, kiovic\}@gmail.com
}

\begin{abstract}
Resumo. EDUTIC es una plataforma de aprendizaje adaptativo con el objetivo de redefinir el aprendizaje móvil, es decir, un modelo de aprendizaje que se amolda a las necesidades, modos de resolver problemas y jerarquía de elementos que se asemejan a las características de cada estudiante de forma inteligente, analizando la información que brinda el uso de la plataforma y generando una retroalimentación para poder sugerir los contenidos que se adapten a los requerimientos educativos individuales. La plataforma gestiona los contenidos de cada institución, por materias y divididos en sesiones.

La plataforma se ofrece como un servicio alojado en la nube que podrá ser contratado por las instituciones educativas y distribuido a todos sus estudiantes. EDUTIC (WWW.edutic.me) es una entidad intuitiva inteligente y sorprendentemente divertida, que mejorara los procesos pedagógicos virtuales disponible en cualquier momento y lugar, utilizando lo ultimo en Inteligencia Artificial, Natural Language Processing y Machine Learning en educación.
\end{abstract}

\section{Escenarios de Uso}

La educación está cambiando, está experimentando un momento disruptivo el cual supone un cambio en su historia debido a las nuevas tecnologías, el uso de dispositivos móviles, dektops y demás relacionados. Uno de los retos de la educación es conseguir que alumnos y profesores estén capacitados para trabajar colaborativamente y aprender a desarrollar óptimamente sus capacidades, desde la etapa escolar, de tal manera poder trabajar en una educación modelo 3.0 que une a padres, institución educativa y estudiantes.

Dichos actores presentan problemas individuales como agentes clave en la educación. Encontramos la falta de educación personalizada para los estudiantes, que ayuden a potencializar sus capacidades, instituciones enfocadas en procesos y no en nuevas metodologías de aprendizaje que les brinden información y retroalimentación a tiempo real de sus estudiantes y profesores, y padres poco informados y con poca orientación con respecto a la educación que recibieron, reciben y deben recibir sus hijos día a día.

El problema se resume en el deficiente aprendizaje de los estudiantes en los colegios y la poca conexión que existe entre los agentes clave de la educación (estudiantes, institución/profesores y padres), lo cual genera una oportunidad de nuevos mercados para la empresa, creando tecnología para dar solución global usando análisis big data y analíticas web, permitiendo ampliar nuestros servicios y a su vez impactar en la competitividad de la empresa, sector y país. 


\section{CBIE-LACLO 2015}

Anais dos Workshops do IV Congresso Brasileiro de Informática na Educação (CBIE 2015)

El problema de la integración de agentes educativos y el deficiente aprendizaje han sido causados por las escasas herramientas educativas a la medida de los estudiantes y al poco uso de tecnología móvil y web óptima en la educación, es así, que cada uno de sus agentes presenta causas, como:

- Estudiantes: La variedad de recursos educativos (libros, textos, videos, y demás) no son personalizados para cada estudiante, pues todos los estudiantes se desarrollan a diferentes velocidades y con diferente ritmo, sin embargo, todo el contenido digital online de aprendizaje ofrece información de la misma manera.

- Padres: La poca información que reciben respecto al desarrollo de sus hijos y sus capacidades, es así, que la elección respecto a que material educativo puede servir mejor y en cada momento, se torna más difícil.

- Institución/Profesores: Igual educación, brindando material educativo que al estudiante no le gusta ni lo motiva y el corto tiempo para una correcta evaluación educativa.

El problema de la integración de agentes educativos y el deficiente aprendizaje, generan diversas consecuencias en cada uno de sus agentes, como:

- Estudiantes: Estudiantes con un desarrollo incompleto de sus competencias, capacidades no aprovechadas, frustraciones y demás relacionados.

- Padres: Padres que cuentan con poca información del desarrollo académico y falta de orientación con respecto a los contenidos que son adecuados para sus hijos.

- Institución/Profesores: La institución no puede brindar un servicio acorde a las necesidades del mercado y los profesores no pueden potencializar todas las capacidades de los estudiantes. En conclusión, el problema será persistente hasta que no se encuentre una alternativa de solución en el país, que puede aprovechar la oportunidad de mercado de educación digital, y si no creamos tecnología en el país, será ofrecida por otro países que nuevamente ampliarán las brechas educativas que tenemos con otros países.

\section{Desarrollo}

En el proceso de diseño de sistemas donde el conocimiento de Interacción Humano Computador (IHC), principios y métodos juegan un papel importante en todas las decisiones de diseño, se debe considerar guías de diseño de interfaces humano computador para aplicaciones de software educativo, considerando la retroalimentación en todo momento, así como la correcta ubicación de los elementos, además se debe proporcionar un ambiente amigable e interactivo $(0,0)$.

La adaptación del proceso de aprendizaje requiere una fase de análisis, previa al desarrollo del proceso, y una fase de aplicación durante el mismo. La primera, partiendo del reconocimiento de la diversidad de los alumnos, consiste en el análisis sobre la forma en que influyen dichas diferencias en el proceso de aprendizaje y de las posibilidades de manipulación que ofrecen. La información recopilada en esta fase de análisis previo permite definir las estrategias de adaptación que se llevarán a cabo en la fase de aplicación. Durante el desarrollo del proceso, determinar con la máxima precisión posible la situación concreta de cada alumno en un instante determinado, es un requisito crítico para poder ofrecerle las opciones más adecuadas para potenciar los efectos positivos del proceso $(0$, 『). 
CBIE-LACLO 2015

Anais dos Workshops do IV Congresso Brasileiro de Informática na Educação (CBIE 2015)

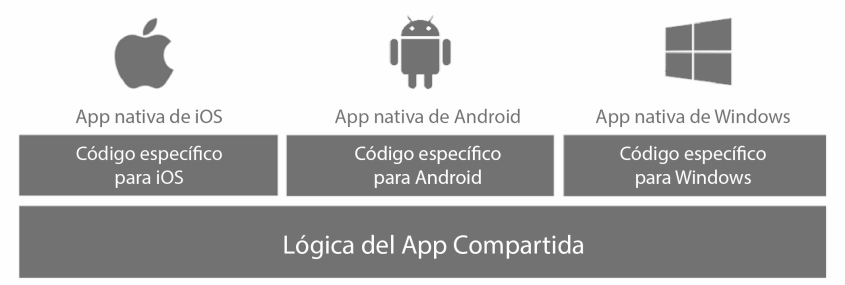

Figura 1. Aplicación del patrón de diseño MVVM para código compartido

Fuente: Elaboración propia.

\subsection{Arquitectura del modelo}

El mundo de los dispositivos móviles está muy dividido debido a la competencia existente entre diversos fabricantes para hacerse con el mercado. La gran variedad y características de los dispositivos móviles llevan a dificultades en el desarrollo de una misma aplicación en diferentes plataformas. Bajo estas premisas, un paso básico para lograr la adaptabilidad y portabilidad de una aplicación es el empleo de un lenguaje de programación estandarizado (0, ).

En este contexto, se propone un framework para la distribución de contenidos que ofrecerá portabilidad en el despliegue de aquellas aplicaciones desarrolladas bajo el mismo. Para alcanzar este objetivo se empleó Xamarin, un kit para el desarrollo de software que permite la construcción de aplicaciones con interfaces de usuario nativas y el uso de código compartido entre diferentes plataformas empleando C\# como lenguaje de programación. Xamarin permite la reutilización de código y ofrece gran compatibilidad, así como la reutilización de librerías escritas en cualquier lenguaje de la plataforma .NET. Teniendo en cuenta la cobertura que se quiere lograr con el Framework de contenidos digitales interactivos, se consideró un desarrollo para las plataformas móviles táctiles más importantes, es así que el cliente fue desarrollado para las plataformas Windows 8.1, iOS y Android.

\subsubsection{Arquitectura de Xamarin}

Xamarin proporciona acceso al $100 \%$ de las APIs nativas disponibles para cada plataforma móvil, asegurando que los desarrolladores no se limitan a un subconjunto arbitrario de características. Los enlaces de datos en el lenguaje C\# proporcionan un mapeo 1 a 1 a las API nativas, al tiempo que añade soporte para características de .Net como LINQ, delegates, eventos y otras ventajas propias del lenguaje, ver Figura 1

Modelo de arquitectura propuesto: Se basa en una arquitectura simple, se puede mantener esta capa de acceso a datos utilizando Windows Azure, de esta forma el consumo de servicios y la comunicación en cada plataforma es indiferente y sencillo como se puede observar en la siguiente la Figura 2 .

\section{Presentación del software}

Creamos una PLATAFORMA de APRENDIZAJE ADAPTATIVO, usando un modelo de aprendizaje que se amolde a las necesidades, modos de resolver problemas y jerarquía de 
CBIE-LACLO 2015

Anais dos Workshops do IV Congresso Brasileiro de Informática na Educação (CBIE 2015)

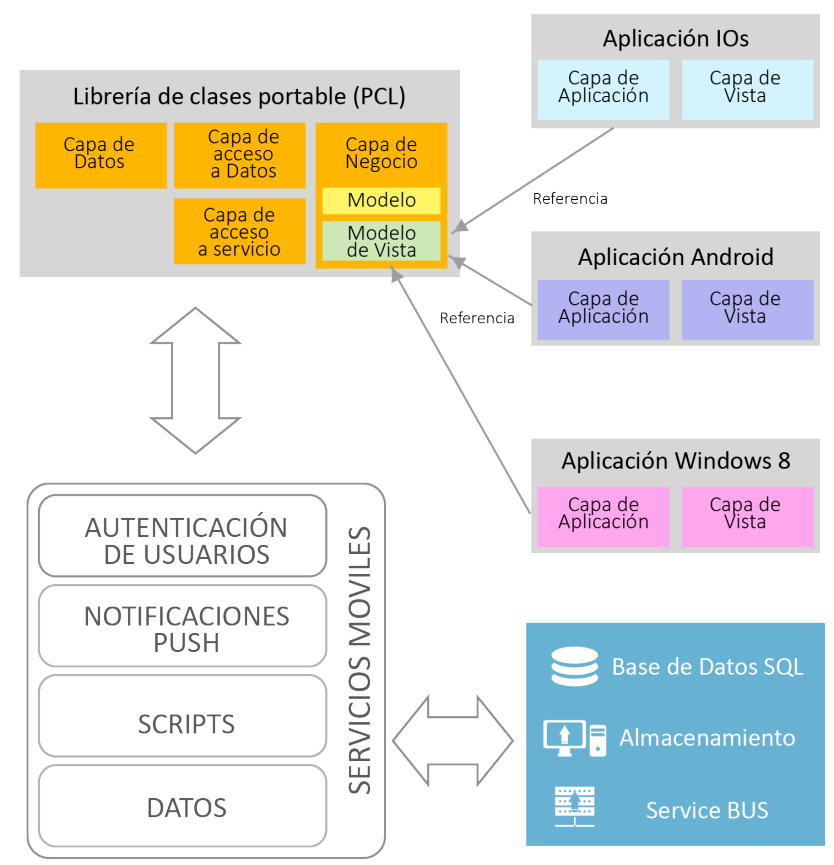

Figura 2. Modelo en capas del modelo propuesto Fuente: Elaboración propia.

elementos que se asemejan a las características de cada estudiante de forma inteligente, analizando la información que brinda el uso de la plataforma y generando una retroalimentación para poder sugerir contenidos que se adapten a los requerimientos educativos individuales.

En el primer piloto aplicado para el desarrollo de la LECTO-ESCRITURA, es decir, desarrollamos un modelo que permita el desarrollo cognitivo de los estudiantes, tomando en cuenta el enfoque comunicativo textual y el desarrollo del pensamiento crítico ligado estrechamente a los niveles de comprensión lectora. Ver más en la Figura 3

\subsection{Cliente EDUTIC}

El cliente de la plataforma EDUTIC es una aplicación de software para dispositivos móviles mediante las cuales se accede a las distintas unidades y herramientas. El acceso se gestiona a través de una lógica de perfiles de usuario, que permite la personalización de los contenidos y herramientas funcionalidades dependiendo del tipo de usuario, ya sea profesor a alumno.

\subsubsection{Navegación e interfaz funcional}

Entre las características de navegación y funcionalidad de las unidades EDUTIC, estas fueron diseñadas para ser compatibles con la navegación en dispositivos móviles, y compatible con las distintas versiones y tamaños de los mismos, es decir navegación e interacción multiplataforma basada en pantallas táctiles. 


\section{CBIE-LACLO 2015}

Anais dos Workshops do IV Congresso Brasileiro de Informática na Educação (CBIE 2015)

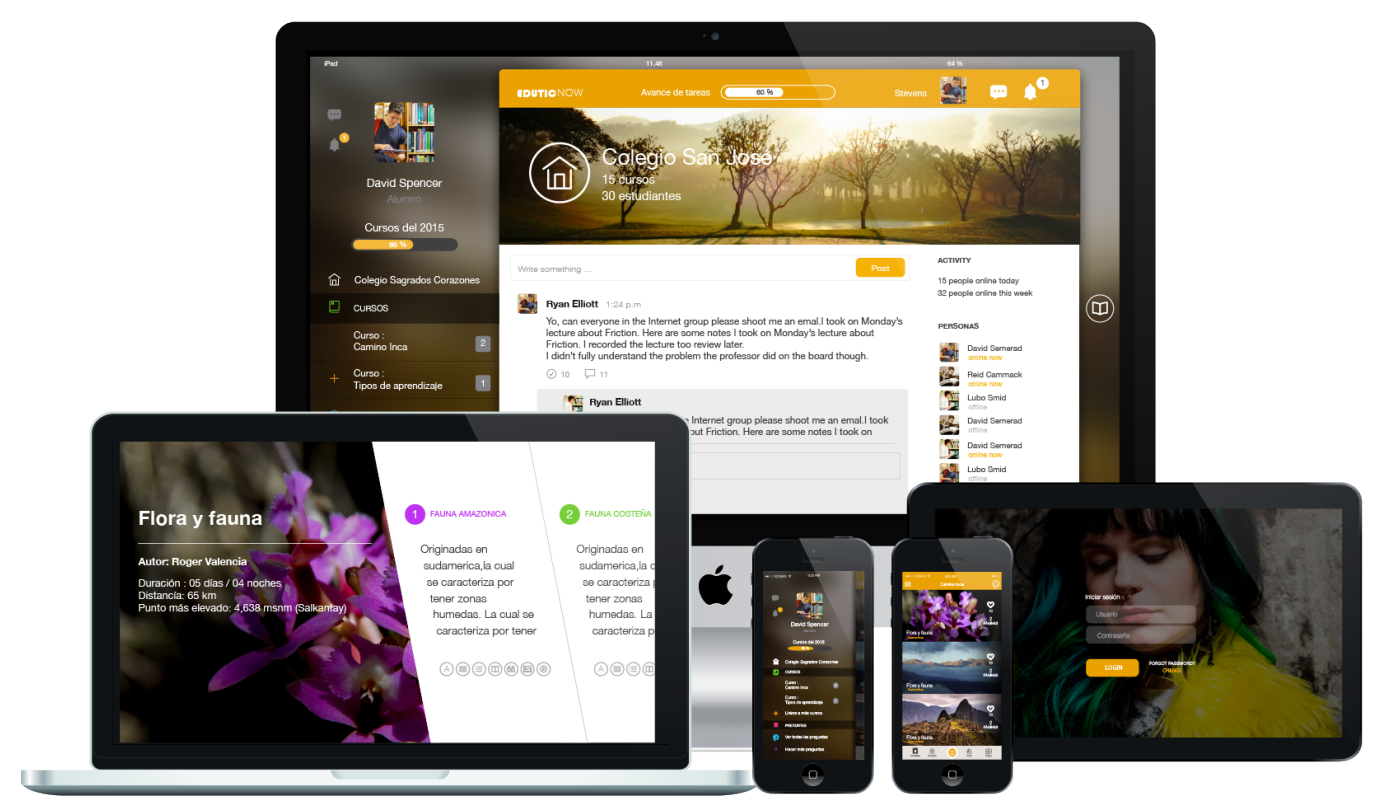

Figura 3. Modelo propuesto en www.edutic.me

Fuente: Elaboración propia.

- Pantallas de organización de secciones

Si el usuario y contraseña ingresados son correctos, se mostrará un muro de objetos de aprendizaje dependiendo del usuario. El usuario podrá visualizar su objetos de aprendizaje teniendo la opción de seleccionarlo, y así poder obtener toda la información correspondiente a este.

- Ubicación y navegación

Después del inicio de sesión, al usuario se le muestra en su muro personal, círculos de estudio en el que está inscrito, y dependiendo de sus circulo se puede visualizar los objetos de aprendizaje asignados a este, además de una lista de tareas pendientes o completadas al cual pertenece, de objetos de aprendizaje asignados, en el cual encontraremos usuarios conectados al sistema, que objetos de aprendizaje han sido asignados y cual son sus tareas respectivas (en estado completo o pendiente). Para visualizar la información completa del objeto de aprendizaje, se tendría que seleccionar la opción abrir.

\subsection{Servicios EDUTIC}

En esta seccion observamos los servicios que brinda la plataforma web de EDUTIC, para la administracion de los contenidos y los usuarios que acceden o crean los mismos (WWW . edutic.me).

\subsubsection{Servidor de contenidos}

Este servicio es responsable del almacenamiento, gestión y administración de las distintas unidades EDUTIC de las editoriales para las distintas asignaturas y niveles educativos del sistema de educacion sonbre el cual se esta trabajando. 
CBIE-LACLO 2015

Anais dos Workshops do IV Congresso Brasileiro de Informática na Educação (CBIE 2015)

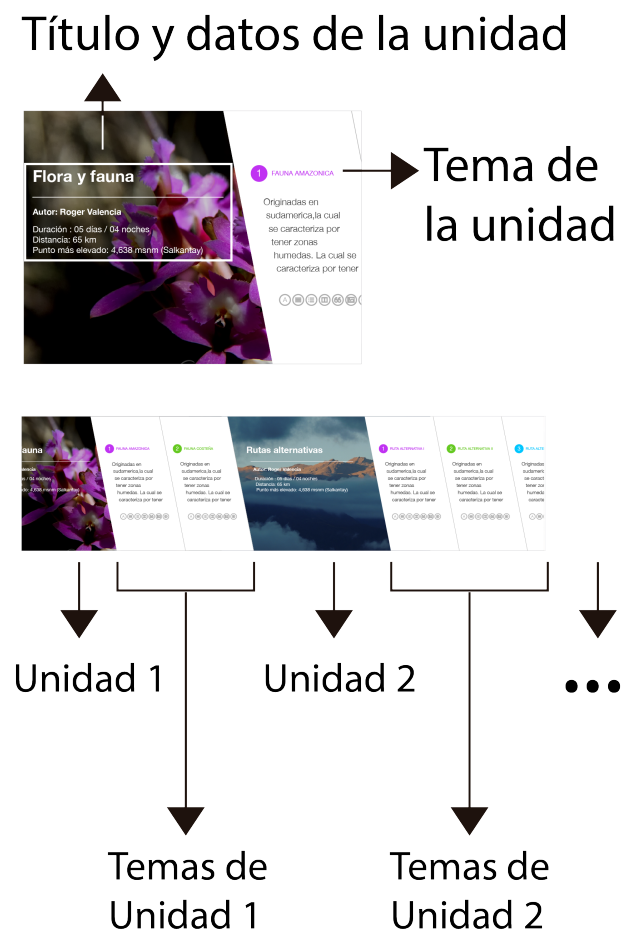

Figura 4. Organizacion de secciones Fuente: Elaboración propia.

La principal particularidad que ofrece el Servidor donde se encuentran los Contenidos, es que rompe con el modelo tradicional de publicación de contenidos educativos, permitiendo la publicación de contenidos en un esquema modular, que en este caso corresponde a las unidades curriculares definidas por los mismos profesores para el avance en las materias indicadas.

\subsubsection{Servidor de Usuarios}

El servidor de Usuarios es el encargado de gestionar y dar mantenimiento a la información y privilegios de los distintos tipos de usuarios definidos en la plataforma, en este caso Alumnos y profesores. Para esto el servidor lleva a cabo tareas de mentenimiento de las entidades que participan, mediante el modelo CRUD (Create, Read, Update and Delete).

\subsubsection{Control de acceso a usuarios}

El control de acceso al software EDUTIC para su versión movil y versión Web se realiza considerando dos etapas :

- Un proceso de autenticación de usuarios y

- Un proceso de autorización en donde el sistema verifica cuales son los privilegios del usuario para acceder a los contenidos y herramientas funcionales desde su cliente EDUTIC. 


\section{Consideraciones finales}

Se presento un análisis sobre el diseño y desarrollo de aplicaciones de libros digitales abiertos, como aplicaciones m-learning, a fin de visualizar el alcance que tiene desde el punto de vista pedagógico y tecnológico para la creación de aplicaciones educativas móviles. Se considero tópicos de IHC, diseño centrado en el usuario, desarrollo multiplataforma y la metodología de desarrollo MEiSE como base para el desarrollo de los casos de estudio. Siguiendo la propuesta presentada se busca mejorar los esfuerzos y establecer una metodología en la creación de materiales educativos, los cuales son bajos debido a su complejidad y elevados costos de producción.

A partir de los resultados obtenidos de la evaluación por expertos se está trabajando en las mejoras del los Objetos de Aprendizaje. El proceso consistirá en un refinamiento iterativo del Objetos de Aprendizaje. A partir de ello, este producto mejorado estará dispuesto a ser evaluado por los estudiantes y docentes de la educación básica regular.

Los contenidos presentados tienen alta demanda cognitiva para los estudiantes, para el caso del libro interactivo orientado al desarrollo de capacidades y en el caso de la historieta al desarrollo de los niveles de comprensión lectora, estos aspectos son requisitos que se tomaron en cuenta para elaborar dichas aplicaciones.

\section{Referências}

Pachler, N., Bachmair , B., \& Cook, J. (2010). Mobile Learning: Structures, Agency, Practices. London: Springer.

Yiqun Li, Aiyuan Guo, Jimmy Addison Lee, \& Gede Putra Kusuma Negara. 2013. A platform on the cloud for self-creation of mobile interactive learning trails. Int. J. Mob. Learn. Organ. 7, 1 (January 2013), 66-80. DOI=10.1504/IJMLO.2013.051574

Johnson, L., Levine, A., Smith, R., 2010. The 2010 Horizon Report. The New Media Consortium, Austin, Texas.

Educause Center for Applied Research [ECAR]. (2010). Study of Undergraduate Students and Information Technology Research Study, 6. Boulder, CO: Educause Center for Applied Research.

Fezile Ozdamlia, Nadire Cavusb (2011). Basic elements and characteristics of mobile learning. Procedia - Social and Behavioral Sciences; Volume 28; Pages 937-942.

N. Cavus (2011), Investigating mobile devices and LMS integration in higher education: Student perspectives, ;presented at Procedia CS, 2011, pp.1469-1474.

Castillo, S. \& Ayala G. (2008). A personalization model for learning objets in mobile learning envireonments. 16th international conference on computers in education (ICCE 2008), Taiwan.

Patokorpi, E., Tetard, F., Qiao, F., Sjovall, N. (2007) Learning objects to Support Constructivist Learning. Chapter 7, Applications, Implications \& Future Directions. Santa Rosa California: Informing Sciences Press. Pp. 187-221

Sergio Martin, Gabriel Diaz, Inmaculada Plaza, Elena Ruiz, Manuel Castro, \& Juan Peire (2011). State of the art of frameworks and middleware for facilitating mobile and ubiquitous learning development. J. Syst. Softw. 84, 11.

Villoria Sáez, A., Martin Pérez, G. \& De las Cuevas Caminero, J. M. (2010). Juegos educativos en dispositivos móviles. Proyecto de sistemas informáticos, Facultad de Informática. Universidad Complutense de Madrid. 
CBIE-LACLO 2015

Anais dos Workshops do IV Congresso Brasileiro de Informática na Educação (CBIE 2015)

Amy Bruckman y Alisa Bandlow (2002) HCI for Kid . Published in The HumanComputer Interaction Handbook. Georgia Institute of Technology

Inkpen, K. (1997). Three Important Research Agendas for Educational Multimedia: Learning, Children and Gender. Paper presented at the Proceedings of Graphics Interface, Calgary, AB.

Alejandro Catala Bolós. (2012). Towards educational places for action, discussion and reaction to support creative learning on interactive surfaces. PHD THESIS, 2012. Universidad Politecnica de Valencia.

Luis Alfaro Casas, Jose Herrera Quispe, Rafael Chaucca (2006) Model for the Customization of an e-Learning System, using Case Based Reasoningand Learning Styles 11/2006, XIX IFIP World Computer Congress (WCC) - Chile 2006.

Ricardo A. Gómez, Alvaro H. Galvis, Olga . (1998). Ingenieria De Software Educativo Con Modelaje Orientado Por Objetos: Un Medio Para Desarrollar Micromundos Interactivos

Abud, F. (2009). MeISE: Metodología de Ingeniería de Software Educativo. Revista Internacional de Educación en Ingeniería.

Granollers, T. (2004). Una metodología que integra la Ingeniería del Software, la Interacción Persona Ordenador y la Accesibiiad en el contexto de equipos de desarrollo multidisciplinares. Tesis Doctoral.Departament de lenguages i Sistemas . 